\title{
Surgical management of camptocormia in Parkinson's disease: systematic review and meta-analysis
}

\author{
Andrew K. Chan, MD, ${ }^{1}$ Alvin Y. Chan, MD, ${ }^{2}$ Darryl Lau, MD, ${ }^{1}$ Beata Durcanova, BS, ${ }^{1}$ \\ Catherine A. Miller, MD, ${ }^{1}$ Paul S. Larson, MD, ${ }^{1}$ Philip A. Starr, MD, PhD, ${ }^{1}$ and \\ Praveen V. Mummaneni, MD ${ }^{1}$
}

1Department of Neurological Surgery, University of California, San Francisco, San Francisco, California; and 2Medical College of Wisconsin, Milwaukee, Wisconsin

\begin{abstract}
OBJECTIVE Camptocormia is a potentially debilitating condition in the progression of Parkinson's disease (PD). It is described as an abnormal forward flexion while standing that resolves when lying supine. Although the condition is relatively common, the underlying pathophysiology and optimal treatment strategy are unclear. In this study, the authors systematically reviewed the current surgical management strategies for camptocormia.
\end{abstract}

METHODS PubMed was queried for primary studies involving surgical intervention for camptocormia in PD patients. Studies were excluded if they described nonsurgical interventions, provided only descriptive data, or were case reports. Secondarily, data from studies describing deep brain stimulation (DBS) to the subthalamic nuclei were extracted for potential meta-analysis. Variables showing correlation to improvement in sagittal plane bending angle (i.e., the vertical angle caused by excessive kyphosis) were subjected to formal meta-analysis.

RESULTS The query resulted in 9 studies detailing treatment of camptocormia: 1 study described repetitive trans-spinal magnetic stimulation (rTSMS), 7 studies described DBS, and 1 study described deformity surgery. Five studies were included for meta-analysis. The total number of patients was 66 . The percentage of patients with over $50 \%$ decrease in sagittal plane imbalance with DBS was $36.4 \%$. A duration of camptocormia of 2 years or less was predictive of better outcomes (OR 4.15).

CONCLUSIONS Surgical options include transient, external spinal stimulation; DBS targeting the subthalamic nuclei; and spinal deformity surgery. Benefit from DBS stimulation was inconsistent. Spine surgery corrected spinal imbalance but was associated with a high complication rate.

https://thejns.org/doi/abs/10.3171/2018.4.JNS173032

KEYWORDS sagittal bending; camptocormia; deep brain stimulation; repetitive trans-spinal magnetic stimulation; spine surgery; functional neurosurgery

$\mathrm{C}$ AMPTOCORMia, an abnormal uncontrollable forward flexion of the spine while standing or walking that corrects while lying flat, is relatively common in patients with Parkinson's disease (PD). The incidence of camptocormia in PD is approximately $6.9 \% .^{16,47}$ Camptocormia is often debilitating and can result in the inability to ambulate or perform activities of daily living. ${ }^{49}$ Nonsurgical treatments include plaster corsets, levodopa, and botulinum neurotoxin or lidocaine injections, though the efficacy of these methods is unclear and outcomes can vary widely between patients. ${ }^{45}$ Although medical therapy is considered first-line therapy for camptocormia in PD, many cases of camptocormia are ultimately refractory to medications alone ${ }^{47}$ For well-selected patients with camptocormia that is not adequately controlled by conservative management strategies, surgery may be indicated. Repetitive trans-spinal magnetic stimulation (rTSMS), deep brain stimulation (DBS), and spinal deformity surgery are surgical options, although the optimal intervention remains unclear.

To this end, we systematically reviewed the available

ABBREVIATIONS DBS = deep brain stimulation; GPi = globus pallidus interna; PD = Parkinson's disease; rTSMS = repetitive trans-spinal magnetic stimulation; STN = subthalamic nucleus.

SUBMITTED December 5, 2017. ACCEPTED April 2, 2018.

INCLUDE WHEN CITING Published online September 14, 2018; DOI: 10.3171/2018.4.JNS173032. 


\begin{tabular}{l|l}
\multicolumn{1}{c}{$\begin{array}{l}\text { PubMed } \\
107 \text { Studies }\end{array}$} \\
$\begin{array}{l}\text { Inclusion Criteria } \\
\text { (see Methods) }\end{array}$ \\
$\begin{array}{ll}54 \text { excluded by title } \\
\text { 48 Full Text Reviewed }\end{array}$ \\
$\begin{array}{l}4 \text { excluded due to case report } \\
15 \text { excluded due to review article } \\
11 \text { excluded due to descriptive } \\
6 \text { excluded due to unrelated } \\
3 \text { excluded due to nonsurgical intervention }\end{array}$
\end{tabular}

\section{Included Studies}

FIG. 1. Flowchart summarizing the study selection.

surgical options for camptocormia in PD patients and associated outcomes. As a secondary aim, we conducted a meta-analysis of DBS treatment in PD patients with camptocormia to investigate potential predictors of successful outcomes.

\section{Methods}

\section{Systematic Review}

We searched PubMed with the following search terms: "Parkinson's disease" and "camptocormia" in October 2017 (Fig. 1). Filters were used to include only articles involving human subjects and written in English. References of reviewed studies were examined for potential additional studies. The query resulted in 107 articles. Articles were excluded if they met any of the following criteria: 1) case report (in attempt to minimize publication bias, though some case reports were included as examples in the discussion); 2) review; 3) the article was not relevant; 4) the intervention was nonsurgical. Potential study biases were assessed and described when appropriate. Level of evidence was assessed for each study and criteria were based on previous methodology. ${ }^{10}$ Level of evidence was determined by the following criteria: 1) Level 1, randomized controlled trials; 2) Level 2, prospective observational or case-controlled studies; 3) Level 3, retrospective studies; 4) Level 4 , case reports or series ( $<10$ patients). The literature search and study design adhered to preferred reporting items for systematic reviews and meta-analyses (PRISMA) guidelines. ${ }^{29}$

\section{Meta-Analysis of DBS Outcomes}

Studies describing only DBS therapy targeting the subthalamic nuclei from the systematic review were included in the meta-analysis if they met the following criteria: 1) minimum 6-month average follow-up; 2) clear quantitative sagittal bending angle measurements before and after DBS unit implantation; 3) studied 4 patients at minimum; 4) DBS was the only surgical intervention. Studies of DBS targeting the globus pallidus interna (GPi) were excluded to minimize confounding. Multiple variables, including age, sex, PD duration, camptocormia duration, and preoperative and postoperative sagittal bending angles, were collected.

\section{Data Collection}

The primary outcome measure was change in sagittal plane bending angle, which was dichotomized into either 1) a decrease of $\leq 15^{\circ}$ versus $>15^{\circ}$ or 2) a decrease of $\leq 50 \%$ versus $>50 \%$. The following variables were collected if they could be unambiguously associated with a change in sagittal bending angle: age, sex, PD duration, camptocormia duration, and baseline sagittal plane bending angle. Variables were dichotomized for meta-analysis to look for possible predictors of outcomes, which is methodology similar to that used in past studies. ${ }^{17,34}$ Age and PD duration were dichotomized into $\leq 60$ or $>60$ years old and $\leq 10$ or $>10$ years, respectively, because the values were approximately characteristic of PD patients who developed camptocormia. ${ }^{9}$ Camptocormia duration was dichotomized into $\leq 2$ or $>2$ years because outcomes have been mixed for PD patients undergoing DBS if their duration of camptocormia was over 2 years. ${ }^{39}$ There was insufficient data to collect appropriate complication rates.

\section{Statistical Analysis}

Sagittal plane bending angle changes were dichotomized (i.e., $\leq 15^{\circ}$ vs $>15^{\circ} ; \leq 50 \%$ reduction vs $>50 \%$ reduction) and stratified by each variable via preliminary statistical analysis, which helped to identify variables potentially associated with outcomes. Measurements were done via either standing films or lateral photos. Student ttests and chi-square tests were used to evaluate continuous and categorical data, respectively. Factors demonstrating a potential association with sagittal plane bending angle changes (i.e., a significance level of $\mathrm{p}<0.05$ ) after preliminary analysis were subjected to formal meta-analysis. Cochran's Q and $\mathrm{I}^{2}$ tests evaluated for study heterogeneity to determine whether a fixed or random effects model was appropriate. Mantel-Haenszel tests calculated odds ratios and $95 \%$ confidence intervals. Potential bias was assessed by visualizing odds ratio funnel plot asymmetry. Wizard Pro 1.8.28 was used for preliminary statistical analysis and Review Manager v5.3 (Nordic Cochrane Centre, Rigshospitalet, 2008) was used for meta-analysis.

\section{Results}

A total of 96 studies were excluded (5 based on the title; 54 based on review of the abstract; and 39 based on full-text review), yielding 9 included studies (Table 1): 1 of these studies described trans-spinal magnetic stimulation, ${ }^{2} 7$ studies described DBS implantation targeting the subthalamic nucleus (STN), ,11,35,36,39,48,52 and 1 study described deformity surgery. ${ }^{50}$

\section{DBS Meta-Analysis Results}

Five studies were included for meta-analysis. ${ }^{3,35,36,39,52}$ Two of the studies listed in Table 1 were excluded. ${ }^{11,48}$ There were no asymmetrical funnel plots. The prelimi- 
TABLE 1. Summary of studies describing surgical intervention for camptocormia in patients with PD

\begin{tabular}{|c|c|c|c|c|c|}
\hline Authors \& Year & $\begin{array}{l}\text { No. of } \\
\text { Cases }\end{array}$ & Study Type & $\begin{array}{c}\text { Evidence } \\
\text { Level }\end{array}$ & Intervention & Result \\
\hline Arii et al., 2014 & 37 & $\begin{array}{l}\text { Randomized sham } \\
\text { controlled trial }\end{array}$ & 1 & Single trial of rTSMS & $\begin{array}{l}\text { Transient avg decrease of } 10.9^{\circ} \text { in treatment group (no signifi- } \\
\text { cant decrease in sham group) }\end{array}$ \\
\hline Asahi et al., 2011 & 4 & Case series & 4 & Bilat DBS (STN) & $75 \%$ of pts improved SB forward flexion by $\geq 12^{\circ}$ \\
\hline Capelle et al., 2011 & 3 & Case series & 4 & Bilat DBS (STN) & All 3 pts improved by mean of $19 \%$ BFM subscore for trunk \\
\hline Sakai et al., 2017 & 14 & Retrosp obs cohort & 3 & Bilat DBS (STN) & $\begin{array}{l}6 \text { of } 14 \text { pts had } \geq 50 \% \text { reduction in SB angle following DBS } \\
\text { surgery }\end{array}$ \\
\hline Sako et al., 2009 & 6 & Case series & 4 & Bilat DBS (STN) & $\begin{array}{l}\text { Mean preop SB angle decreased by } 78 \% \text { after surgery ( } \geq 5 \text { mos } \\
\text { follow-up) }\end{array}$ \\
\hline $\begin{array}{l}\text { Schulz-Schaeffer et } \\
\text { al., } 2015\end{array}$ & 25 & Retrosp obs cohort & 3 & Bilat DBS (STN) & $\begin{array}{l}13 \text { of } 25 \text { ( } 52 \%) \text { pts had improvement of SB angle by } \geq 50 \% \\
\text { ( } \geq 6 \text { mos follow-up) }\end{array}$ \\
\hline Umemura et al., 2010 & 8 & Case series & 4 & Bilat DBS (STN) & 4 of 8 pts improved short term; 5 of 8 pts improved long term \\
\hline Yamada et al., 2015 & 17 & Prosp trial & 2 & Bilat DBS (STN) & $\begin{array}{l}\text { Avg preop SB angle decreased significantly at } 3 \text { mos follow-up } \\
(-40.7 \%) \& \text { last follow up }(-34.8 \%)\end{array}$ \\
\hline Wadia et al., 2011 & 2 & Case series & 4 & Spinal fixation & $\begin{array}{l}\text { Subjective improvement of back pain following T3 to pelvis fixa- } \\
\text { tion w/ or w/o osteotomy at last follow-up }\end{array}$ \\
\hline
\end{tabular}

Avg = average; BFM = Burke-Fahn-Marsden; obs = observational; prosp = prospective; $p$ ts = patients; retrosp = retrospective; SB = sagittal bending.

nary data are summarized in Table 2 . The total number of patients included was 66 . The percentage of patients who had over a $15^{\circ}$ absolute decrease in sagittal plane bending angle was $51.5 \%$ (34/66). The percentage of patients who had over a $50 \%$ decrease in sagittal plane bending angle was $36.4 \%$ (24/66). The mean sagittal plane bending angles prior to and following DBS surgery were $56.6^{\circ} \pm$ $5.1^{\circ}$ and $38.4^{\circ} \pm 6.6^{\circ}$ respectively. The mean sagittal plane bending angle change following DBS treatment was $-18^{\circ}$ $\pm 6.1^{\circ}$. Shorter camptocormia duration (i.e., $\leq 2$ years) was predictive of a greater than $15^{\circ}$ decrease in sagittal bending angle following bilateral STN DBS (OR 4.15, 95\% CI 1.41-12.18, $\mathrm{p}=0.01$ ). Shorter camptocormia duration (i.e., $\leq 2$ years) and younger age (i.e., $\leq 60$ years) did not predict a $50 \%$ decrease in sagittal plane bending angle (Fig. 2).

\section{Discussion}

Here the current evidence for outcomes of surgical treatment in PD patients with camptocormia is reviewed and summarized, including the present meta-analysis for DBS treatment targeting the STN.

\section{Characteristics of Camptocormia}

Camptocormia is a postural deformity defined as severe forward flexion of the thoracolumbar spine that is

TABLE 2. Summary of preliminary data results

\begin{tabular}{|c|c|c|c|c|c|c|}
\hline \multirow[b]{2}{*}{ Variable } & \multicolumn{3}{|c|}{ SBA Absolute Decrease } & \multicolumn{3}{|c|}{ SBA \% Reduction } \\
\hline & $\leq 15^{\circ}$ & $>15^{\circ}$ & $p$ Value & $\leq 50 \%$ & $>50 \%$ & $\mathrm{p}$ Value \\
\hline Age, mean \pm SD & $65.2 \pm 3.3$ & $61.7 \pm 4.1$ & 0.192 & $66.0 \pm 2.4$ & $56.8 \pm 5.8$ & $<0.001^{*}$ \\
\hline Sex & & & 0.482 & & & 0.812 \\
\hline Male & 18 & 22 & & 25 & 15 & \\
\hline Female & 14 & 12 & & 17 & 9 & \\
\hline PD duration & & & 0.484 & & & 0.099 \\
\hline$\leq 10 \mathrm{yrs}$ & 7 & 10 & & 8 & 9 & \\
\hline$>10 \mathrm{yrs}$ & 25 & 24 & & 34 & 15 & \\
\hline Camptocormia duration & & & $<0.002^{*}$ & & & $0.002^{*}$ \\
\hline$\leq 2 \mathrm{yrs}$ & 6 & 16 & & 10 & 12 & \\
\hline$>2$ yrs & 26 & 12 & & 32 & 6 & \\
\hline Baseline TL angle & & & 0.478 & & & 0.672 \\
\hline$\leq 45^{\circ}$ & 15 & 13 & & 17 & 11 & \\
\hline$>45^{\circ}$ & 17 & 21 & & 25 & 13 & \\
\hline
\end{tabular}




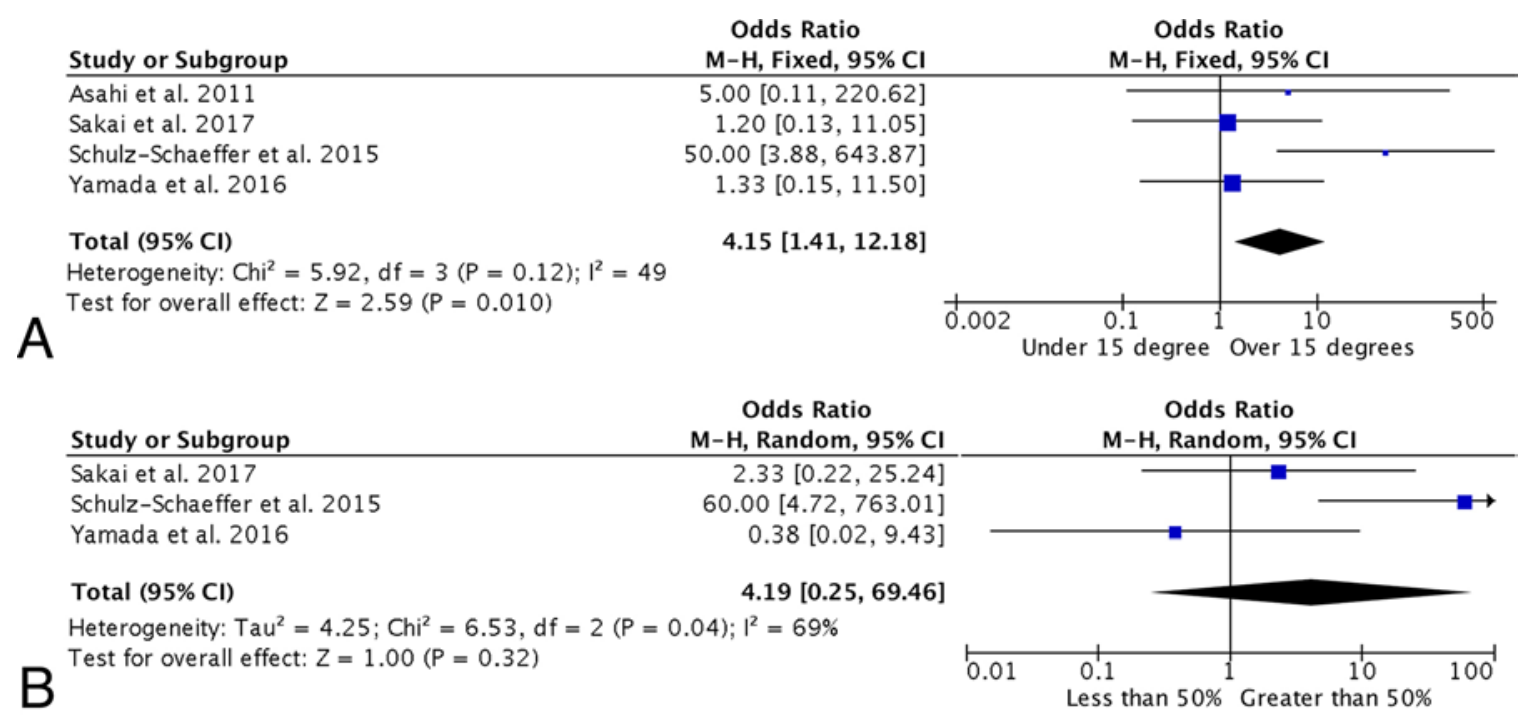

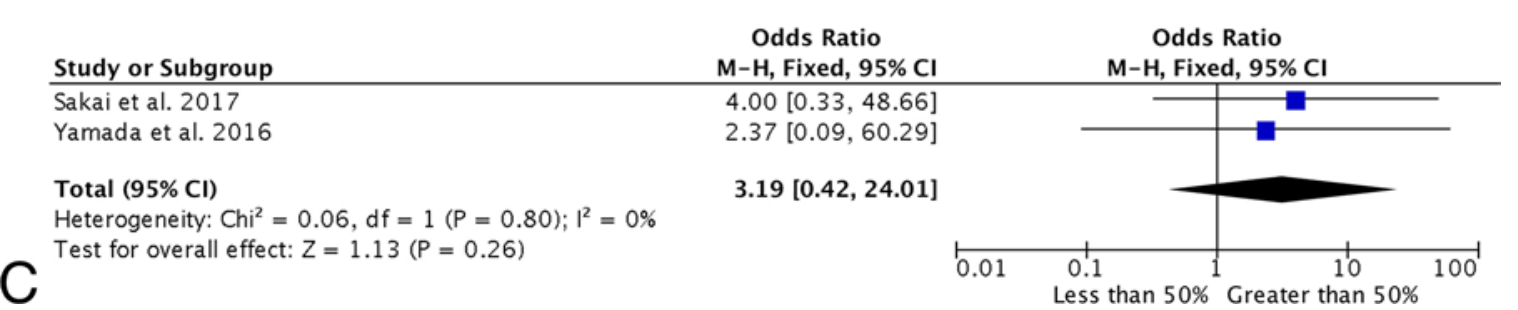

FIG. 2. Meta-analyses examining factors associated with improvement of camptocormia. $A: \leq 2$ years over $>2$ years camptocormia duration with respect to over $15^{\circ}$ thoracolumbar $(T L)$ angle decrease. $\mathbf{B}: \leq 2$ years over $>2$ years camptocormia duration with respect to $>50 \%$ TL angle decrease. $C: \leq 60$ years old over $>60$ years old with respect to $>50 \%$ TL angle decrease. A significant predictor of $>15^{\circ} \mathrm{TL}$ angle decrease $(p<0.05)$ after meta-analysis was $\leq 2$ years over $>2$ years camptocormia duration $(A)$. $d f=$ degrees of freedom; $\mathrm{M}-\mathrm{H}=$ Mantel-Haenszel. Figure is available in color online only.

apparent during ambulation and improves in the supine position. ${ }^{9}$ There is no universal quantitative threshold to establish a diagnosis of camptocormia, but a widely accepted value is a sagittal plane bending angle greater than roughly $45^{\circ}$ while standing..$^{4,5}$ The deformity generally develops within 10 years of PD onset and responds poorly to levodopa treatment. ${ }^{9,15}$ The development of camptocormia is more likely in PD patients with advanced disease progression, more severe motor symptoms, and relatively high levodopa dose requirements. ${ }^{41,47}$

The pathogenesis of camptocormia is unclear but considered to be multifactorial, with various mechanisms contributing to the overall condition. ${ }^{22,49}$ Although spinal surgery has been attempted to treat camptocormia, primary spinal deformity (rigid kyphoscoliosis) is likely not a primary driver of the condition, as the characteristic flexion disappears in the supine position ${ }^{26}$ and thus we do not recommend spinal surgery as an initial treatment. Moreover, as a result of this positional dependence, the condition has been described as an axial dystonia. ${ }^{9}$ Conversely, PD progression may also lead to regional instability due to a focal myopathy and degeneration of the paraspinal muscles, although the mechanisms of the myopathy and degeneration are unclear. ${ }^{14,24,44}$ Indeed, in a study of 20 PD patients with camptocormia, MRI showed edema and fatty degeneration of the paravertebral muscles, implying a systematic destabilization of lumbar trunk musculature. ${ }^{23}$ Histological analyses support these radiographic findings, as camptocormia musculature reveals structural defects, including myofibrillary disintegration, Z-band streaming, or rod-like structures in the muscular lesions ${ }^{51}$ Further, camptocormia could be related to dopamine depletion as well, considering that levodopa medication can produce a mild improvement in camptocormia in some PD patients. A study of 23 patients showed that camptocormia severity fluctuated over the course of the day and that in $22 \%$ of the patients the condition improved with levodopa treatment. ${ }^{22}$ However, levodopa has only had inconsistent results in patients with PD and camptocormia ${ }^{12}$ and thus low dopamine levels cannot be the only factor involved.

\section{DBS Targeting the Subthalamic Nuclei}

The most common form of surgical therapy used for treating camptocormia in PD patients is bilateral STN DBS. DBS is effective in treating motor symptoms in PD patients, ${ }^{31}$ though improvements in postoperative quality of life have only been shown in patients who are younger than 65 years. ${ }^{13}$ STN DBS may also reverse cognitive decline in PD patients, ${ }^{18,43}$ making DBS an important treatment option for patients whose symptoms are not adequately controlled by noninvasive therapy. The implantation procedure was traditionally done while the patient was awake, but "asleep" DBS surgery (i.e., surgery done under general anesthesia using intraoperative imaging) has been shown to be effective. ${ }^{28,32}$ How STN DBS works is still unclear, 
but the prevailing hypothesis is that stimulation interferes with neuronal circuits and inhibits the production or release of neurotransmitters or hormones. ${ }^{8}$

Recently, there has been more evidence for using STN DBS to treat camptocormia secondary to PD. ${ }^{12}$ For example, a study of 14 PD patients showed that $42.9 \%$ - slightly under half-had at least 50\% improvement in their sagittal plane bending after DBS stimulation. ${ }^{35}$ In contrast, $35.7 \%$ of the patients had no change in sagittal plane bending angle following surgery. Another study of 8 patients showed improvement in only 50\% in the short term and $62.5 \%$ in the long term, although the exact postoperative time course was unclear. ${ }^{48}$ Though STN DBS is widely used for treating $\mathrm{PD}$, there have been no established predictors of success for PD-associated camptocormia. A systematic review of studies describing patients undergoing DBS for the treatment of PD-related camptocormia found that $68 \%$ of patients $(\mathrm{n}=32)$ showed significant improvement following DBS surgery. ${ }^{12}$ The authors concluded that DBS showed benefits but that the role of DBS and the optimal timing of surgery after the onset of camptocormia were unclear. Our analysis reveals that duration of camptocormia may be inversely related to the efficacy of STN DBS stimulation. Specifically, PD patients receiving STN DBS within 2 years of the onset of camptocormia had increased odds of achieving at least a $15^{\circ}$ improvement in their sagittal plane bending angle as compared to patients with longer disease duration.

\section{DBS Targeting the GPi}

The globus pallidus was a target initially described for primary dystonia ${ }^{21}$ but has also been more recently been used to treat camptocormia. ${ }^{20,27,30}$ The GPi has been shown to be an appropriate target for DBS in PD patients to improve motor function. A randomized double-blind controlled trial showed that patients had significant improvement in motor function with either STN or pallidal stimulation, with low complication rates. ${ }^{31}$ A second randomized controlled trial demonstrated that there was no difference between STN and pallidal stimulation in terms of improved motor function for PD patients. ${ }^{19}$ It is posited that potential abnormal asynchronous activity in the globus pallidus medialis disrupts normal posturing via involuntary truncal flexion; ${ }^{30}$ stimulating the GPi is thought to resolve this activity and restore physiological posturing. Supporting this theory, an initial case report demonstrated markedly improved camptocormia in a 49-year-old woman following right pallidotomy. ${ }^{42}$ Further evidence is a case study describing 2 nonambulatory patients with dystonic camptocormia who underwent GPi DBS that allowed them to regain the ability to walk. ${ }^{20}$ However, evidence for GPi DBS in camptocormia is still scarce.

Although GPi DBS can have some efficacy in improving camptocormia secondary to dystonia, the evidence has been limited to mostly case reports for treatment of PD-related camptocormia. ${ }^{27,30,46}$ One case report described a 57-year-old PD patient who developed severe camptocormia and was successfully treated with high-frequency bilateral GPi DBS. ${ }^{46}$ Her camptocormia did not improve after optimization of her medications and thus she underwent asleep DBS unit implantation, which resulted in placement of the leads in the ventrodorsal GPi. Her camptocormia was substantially improved at 2 months and completely resolved at 1 year. Another case involved a 62-yearold PD patient with drug-resistant severe camptocormia that responded well to bilateral pallidal stimulation. ${ }^{27}$ At 14 months following the implantation, his camptocormia had almost completely resolved. Both of these examples described favorable outcomes, but case reports are subject to publication biases and are classified as providing a low level of evidence. Larger, prospective studies are required to determine the efficacy of GPi DBS in camptocormia secondary to $\mathrm{PD}$.

\section{Repetitive Trans-Spinal Magenetic Stimulation}

Repetitive trans-spinal magnetic stimulation (rTSMS) may have a role in improving PD-associated camptocormia. ${ }^{1}$ A randomized placebo-controlled study showed that a single trial of rTSMS resulted in a transient decrease of thoracolumbar spinal flexion angle. ${ }^{2}$ Patients were randomly assigned to either a stimulation group $(n=19)$ or a sham treatment group $(\mathrm{n}=18)$; the 2 groups had average angle decreases of $10.9^{\circ}$ and $-0.1^{\circ}$ ( an increase of $0.1^{\circ}$ ), respectively. The authors speculated that the effect of rTSMS was possibly due to a blockade of afferent sensory nerve fibers or disruption of akinetic corticostriatal activity. There are important limitations of this study. First, patients with sham stimulation did not experience the twitch sensation in their paraspinal muscles as did those undergoing rTSMS. Second, the sample size was relatively small. Last, results were transient.

\section{Predictors of Greater Improvement in Sagittal Plane Bending Angle Following DBS Surgery}

The results from our meta-analysis showed that a shorter camptocormia duration before DBS unit implantation was a significant predictor of a greater postoperative decrease in sagittal bending angle. A study showed that the duration of camptocormia may predict the efficacy of DBS. ${ }^{39}$ The study investigated 25 PD patients with camptocormia and found that 13 patients had at least a $50 \%$ decrease in their sagittal bending angle. Analysis showed that patients with shorter camptocormia duration (e.g., 1.5 years) were more likely than those with longer duration to benefit from DBS. We found a similar result in that camptocormia duration of 2 years or less was predictive of a decreased postoperative sagittal plane bending angle of at least $15^{\circ}$ but camptocormia duration was not predictive of whether patients had at least a $50 \%$ decrease in sagittal plane bending.

\section{Spinal Deformity Surgery}

Evidence is limited with respect to spinal surgery for camptocormia correction. There are a few case reports and short series in the literature. ${ }^{33,49,50}$ Peek et al. described a 55-year-old man who had sagittal plane bending of $45^{\circ}$ at the hip that resolved after lying supine. ${ }^{33}$ The patient initially underwent a T7-to-ilium posterior fixation that required a revision with multiple parallel rods and crosslinks when the posterior fixation rod disconnected from the iliac screw. He presented a few months later with loosening of 


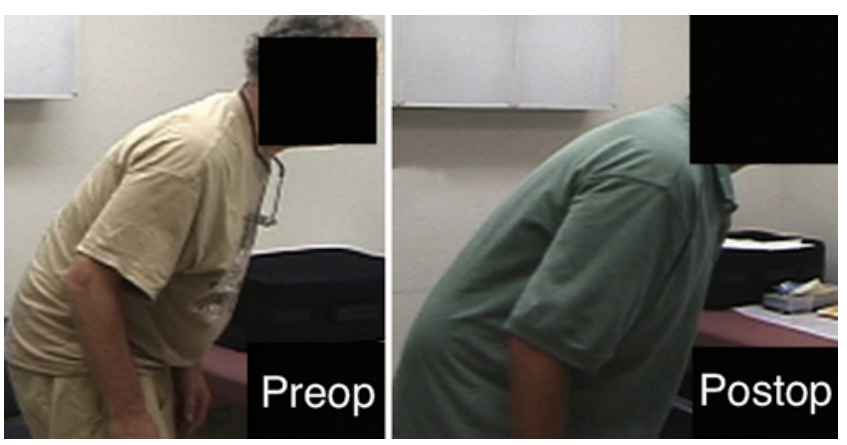

FIG. 3. Left: Preoperative photograph of a 59 -year-old man with advanced idiopathic PD and severe camptocormia who underwent placement of bilateral STN deep brain stimulators and a right chest dual channel pulse generator. Right: Photograph of the patient at 2 years' follow-up. His gait and ambulation had improved, but there was no significant improvement in his camptocormia posture. From Upadhyaya et al: Spinal deformity and Parkinson disease: a treatment algorithm. Neurosurg Focus 28(3):E5, 2010. Used with permission. Figure is available in color online only.

the L5 pedicle screw, which ultimately required a 2-stage revision with multiple interbody fusions. However, the long-term result was favorable, as the physiological sagittal plane bending angle was restored at 1 year after his last operation. The authors suggest that camptocormia can be treated via spinal fixation, but given the potential surgical risks, it may be considered only in the case that DBS and nonsurgical options have failed and the patient has the strong desire to remain ambulatory. Patients should be cautioned that the reoperation rate and complication rate are high.

Spinal fusion surgery for PD patients could be generally unfavorable due to high rates of complications. . $^{6,25,38}$ A retrospective review of 14 PD patients who underwent spine surgery showed that 12 of them required multiple operations (mean 2.6, range 1-11) and had a high rate of complications. ${ }^{6}$ Furthermore, DBS has been shown to be relatively effective for camptocormia, with low rates of morbidity ${ }^{12}$ which is why patients and surgeons may opt against spinal fusion surgery as a first option. Indeed, a proposed algorithm for treating PD patients with camptocormia indicated that spinal surgery should be avoided completely if the patient does not have myelopathy or radiculopathy. ${ }^{49}$ However, DBS may fail to correct camptocormia (Fig. 3) and spinal surgery may be one of the few remaining treatment options. Moreover, the present study's results showed that the longer duration of camptocormia resulted in lower efficacy of DBS for restoring physiological sagittal balance, and thus spinal fusion surgery may be the only remaining option for patients who have had camptocormia for long durations. Further research comparing the efficacy of DBS versus spinal fusion surgery in patients with longstanding camptocormia is necessary to clarify this hypothesis.

\section{Study Limitations}

There are a number of limitations to to this study. First, only a small number of studies met the predetermined criteria. Moreover, the small number of studies used for meta-analysis - the secondary aim of this study-resulted a small sample size, which likely lowered statistical power and increased the potential margin for error. However, we would expect a negative rather than positive result with a small sample size; thus our finding is likely still valid. Further meta-anlayses will be required after more high-quality investigations are conducted. This may help to identify additional factors that portend better outcomes. Second, studies used different methods for measuring the sagittal bending angle; however, potential confounding should have been minimal since the comparisons were made between pre- and postoperative angles rather than between study comparisons. Future work may adopt widely accepted, valid, and reliable measurements to aid in acrossstudy comparisons, such as the Schwab SRS (Scoliosis Research Society) classification system. ${ }^{40}$ Third, there are inherent limitations to meta-analysis, especially in neurosurgery ${ }^{37}$ For example, only published data were available for analysis, and thus the results may be skewed toward more positive results (i.e., publication bias). Still, the funnel plots of odds ratios investigating bias did not reveal asymmetry, reducing the risk of this confounding effect, and the PRISMA guidelines for meta-analyses were followed. Fourth, the majority of studies found in our review did not meet the criteria for level 1 or 2 evidence. Additional highquality studies investigating surgical options for camptocormia are necessary.

\section{Conclusions}

We systematically reviewed the existing literature on surgical treatment in camptocormia. The surgical options include rTSMS, STN DBS, or GPi DBS, and spinal deformity corrective surgery. In general, STN DBS therapy worked for some patients but was inconsistent in terms of benefit. A meta-analysis of available data found that a camptocormia duration of 2 years or less was predictive of a larger decrease in sagittal plane bending following STN DBS therapy. Evidence for GPi DBS is limited to case reports and should be interpreted using appropriate clinical judgment. Furthermore, evidence for spinal fusion surgery to correct sagittal imbalance due to camptocormia was limited, but spinal fusion was associated with a high complication rate in this population. Future directions for possible data collection include the use of large, prospective registries like the Quality Outcomes Database (QOD) - especially with coordination among centers that have both high-volume PD DBS and spinal deformity surgery practice settings-to facilitate larger scale studies and identification of more accurate predictors of outcomes for patients suffering from camptocormia.

\section{References}

1. Akiyama H, Nukui S, Akamatu M, Hasegawa Y, Nishikido O, Inoue S: Effectiveness of spinal cord stimulation for painful camptocormia with Pisa syndrome in Parkinson's disease: a case report. BMC Neurol 17:148, 2017

2. Arii Y, Sawada Y, Kawamura K, Miyake S, Taichi Y, Izumi Y, et al: Immediate effect of spinal magnetic stimulation on camptocormia in Parkinson's disease. J Neurol Neurosurg Psychiatry 85:1221-1226, 2014

3. Asahi T, Taguchi Y, Hayashi N, Hamada H, Dougu N, Takashima S, et al: Bilateral subthalamic deep brain stimulation 
for camptocormia associated with Parkinson's disease. Stereotact Funct Neurosurg 89:173-177, 2011

4. Ashour R, Jankovic J: Joint and skeletal deformities in Parkinson's disease, multiple system atrophy, and progressive supranuclear palsy. Mov Disord 21:1856-1863, 2006

5. Azher SN, Jankovic J: Camptocormia: pathogenesis, classification, and response to therapy. Neurology 65:355-359, 2005

6. Babat LB, McLain RF, Bingaman W, Kalfas I, Young P, Rufo-Smith C: Spinal surgery in patients with Parkinson's disease: construct failure and progressive deformity. Spine (Phila Pa 1976) 29:2006-2012, 2004

7. Baker JF, McClelland S III, Line BG, Smith JS, Hart RA, Ames CP, et al: In-hospital complications and resource utilization following lumbar spine surgery in patients with Parkinson disease: evaluation of the National Inpatient Sample database. World Neurosurg 106:470-476, 2017

8. Benabid AL, Chabardes S, Mitrofanis J, Pollak P: Deep brain stimulation of the subthalamic nucleus for the treatment of Parkinson's disease. Lancet Neurol 8:67-81, 2009

9. Bloch F, Houeto JL, Tezenas du Montcel S, Bonneville F, Etchepare F, Welter ML, et al: Parkinson's disease with camptocormia. J Neurol Neurosurg Psychiatry 77:12231228,2006

10. Burns PB, Rohrich RJ, Chung KC: The levels of evidence and their role in evidence-based medicine. Plast Reconstr Surg 128:305-310, 2011

11. Capelle HH, Schrader C, Blahak C, Fogel W, Kinfe TM, Baezner H, et al: Deep brain stimulation for camptocormia in dystonia and Parkinson's disease. J Neurol 258:96-103, 2011

12. Chieng LO, Madhavan K, Wang MY: Deep brain stimulation as a treatment for Parkinson's disease related camptocormia. J Clin Neurosci 22:1555-1561, 2015

13. Derost PP, Ouchchane L, Morand D, Ulla M, Llorca PM, Barget M, et al: Is DBS-STN appropriate to treat severe Parkinson disease in an elderly population? Neurology 68:13451355,2007

14. Diederich NJ, Goebel HH, Dooms G, Bumb A, Huber F, Kompoliti K, et al: Camptocormia associated with focal myositis in multiple-system atrophy. Mov Disord 21:390-394, 2006

15. Djaldetti R, Mosberg-Galili R, Sroka H, Merims D, Melamed E: Camptocormia (bent spine) in patients with Parkinson's disease-characterization and possible pathogenesis of an unusual phenomenon. Mov Disord 14:443-447, 1999

16. Doherty KM, van de Warrenburg BP, Peralta MC, SilveiraMoriyama L, Azulay JP, Gershanik OS, et al: Postural deformities in Parkinson's disease. Lancet Neurol 10:538-549, 2011

17. Englot DJ, Berger MS, Barbaro NM, Chang EF: Predictors of seizure freedom after resection of supratentorial low-grade gliomas. A review. J Neurosurg 115:240-244, 2011

18. Enrici I, Mitkova A, Castelli L, Lanotte M, Lopiano L, Adenzato M: Deep brain stimulation of the subthalamic nucleus does not negatively affect social cognitive abilities of patients with Parkinson's disease. Sci Rep 7:9413, 2017

19. Follett KA, Weaver FM, Stern M, Hur K, Harris CL, Luo P, et al: Pallidal versus subthalamic deep-brain stimulation for Parkinson's disease. N Engl J Med 362:2077-2091, 2010

20. Hagenacker T, Gerwig M, Gasser T, Miller D, Kastrup O, Jokisch D, et al: Pallidal deep brain stimulation relieves camptocormia in primary dystonia. J Neurol 260:1833-1837, 2013

21. Krauss JK, Yianni J, Loher TJ, Aziz TZ: Deep brain stimulation for dystonia. J Clin Neurophysiol 21:18-30, 2004

22. Lepoutre AC, Devos D, Blanchard-Dauphin A, Pardessus V, Maurage CA, Ferriby D, et al: A specific clinical pattern of camptocormia in Parkinson's disease. J Neurol Neurosurg Psychiatry 77:1229-1234, 2006

23. Margraf NG, Rohr A, Granert O, Hampel J, Drews A, Deus- chl G: MRI of lumbar trunk muscles in patients with Parkinson's disease and camptocormia. J Neurol 262:1655-1664, 2015

24. Margraf NG, Wrede A, Rohr A, Schulz-Schaeffer WJ, Raethjen J, Eymess A, et al: Camptocormia in idiopathic Parkinson's disease: a focal myopathy of the paravertebral muscles. Mov Disord 25:542-551, 2010

25. McClelland S III, Baker JF, Smith JS, Line BG, Hart RA, Ames CP, et al: Complications and operative spine fusion construct length in Parkinson's disease: a nationwide population-based analysis. J Clin Neurosci 43:220-223, 2017

26. Melamed E, Djaldetti R: Camptocormia in Parkinson's disease. J Neurol 253 (Suppl 7):VII14-VII16, 2006

27. Micheli F, Cersósimo MG, Piedimonte F: Camptocormia in a patient with Parkinson disease: beneficial effects of pallidal deep brain stimulation. Case report. J Neurosurg 103:10811083,2005

28. Mirzadeh Z, Chapple K, Lambert M, Evidente VG, Mahant P, Ospina MC, et al: Parkinson's disease outcomes after intraoperative CT-guided "asleep" deep brain stimulation in the globus pallidus internus. J Neurosurg 124:902-907, 2016

29. Moher D, Liberati A, Tetzlaff J, Altman DG: Preferred reporting items for systematic reviews and meta-analyses: the PRISMA statement. PLoS Med 6:e1000097, 2009

30. Nandi D, Parkin S, Scott R, Winter JL, Joint C, Gregory R, et al: Camptocormia treated with bilateral pallidal stimulation. J Neurosurg 97:461-466, 2002

31. Obeso JA, Olanow CW, Rodriguez-Oroz MC, Krack P, Kumar R, Lang AE: Deep-brain stimulation of the subthalamic nucleus or the pars interna of the globus pallidus in Parkinson's disease. N Engl J Med 345:956-963, 2001

32. Ostrem JL, Ziman N, Galifianakis NB, Starr PA, Luciano MS, Katz M, et al: Clinical outcomes using ClearPoint interventional MRI for deep brain stimulation lead placement in Parkinson's disease. J Neurosurg 124:908-916, 2016

33. Peek AC, Quinn N, Casey AT, Etherington G: Thoracolumbar spinal fixation for camptocormia in Parkinson's disease. J Neurol Neurosurg Psychiatry 80:1275-1278, 2009

34. Rowland NC, Englot DJ, Cage TA, Sughrue ME, Barbaro NM, Chang EF: A meta-analysis of predictors of seizure freedom in the surgical management of focal cortical dysplasia. J Neurosurg 116:1035-1041, 2012

35. Sakai W, Nakane S, Urasaki E, Toyoda K, Sadakata E, Nagaishi A, et al: The cross-sectional area of paraspinal muscles predicts the efficacy of deep drain stimulation for camptocormia. J Parkinsons Dis 7:247-253, 2017

36. Sako W, Nishio M, Maruo T, Shimazu H, Matsuzaki K, Tamura T, et al: Subthalamic nucleus deep brain stimulation for camptocormia associated with Parkinson's disease. Mov Disord 24:1076-1079, 2009

37. Sampson JH, Barker FG II: Methodology and reporting of meta-analyses in the neurosurgical literature. J Neurosurg 120:791-794, 2014

38. Sapkas G, Lykomitros V, Soultanis K, Papadopoulos EC, Papadakis M: Spinal surgery in patients with Parkinson's disease: unsatisfactory results, failure and disappointment. Open Orthop J 8:264-267, 2014

39. Schulz-Schaeffer WJ, Margraf NG, Munser S, Wrede A, Buhmann C, Deuschl G, et al: Effect of neurostimulation on camptocormia in Parkinson's disease depends on symptom duration. Mov Disord 30:368-372, 2015

40. Schwab F, Ungar B, Blondel B, Buchowski J, Coe J, Deinlein D, et al: Scoliosis Research Society-Schwab adult spinal deformity classification: a validation study. Spine (Phila Pa 1976) 37:1077-1082, 2012

41. Seki M, Takahashi K, Koto A, Mihara B, Morita Y, Isozumi $\mathrm{K}$, et al: Camptocormia in Japanese patients with Parkinson's disease: a multicenter study. Mov Disord 26:2567-2571, 2011 
42. Sławek J, Derejko M, Lass P: Camptocormia as a form of dystonia in Parkinson's disease. Eur J Neurol 10:107-108, 2003

43. Smeding HM, van den Munckhof P, Esselink RA, Schmand B, Schuurman PR, Speelman JD: Reversible cognitive decline after DBS STN in PD and displacement of electrodes. Neurology 68:1235-1236, 2007

44. Spuler S, Krug H, Klein C, Medialdea IC, Jakob W, Ebersbach $\mathrm{G}$, et al: Myopathy causing camptocormia in idiopathic Parkinson's disease: a multidisciplinary approach. Mov Disord 25:552-559, 2010

45. Srivanitchapoom P, Hallett M: Camptocormia in Parkinson's disease: definition, epidemiology, pathogenesis and treatment modalities. J Neurol Neurosurg Psychiatry 87:75-85, 2016

46. Thani NB, Bala A, Kimber TE, Lind CR: High-frequency pallidal stimulation for camptocormia in Parkinson disease: case report. Neurosurgery 68:E1501-E1505, 2011

47. Tiple D, Fabbrini G, Colosimo C, Ottaviani D, Camerota F, Defazio G, et al: Camptocormia in Parkinson disease: an epidemiological and clinical study. J Neurol Neurosurg Psychiatry 80:145-148, 2009

48. Umemura A, Oka Y, Ohkita K, Yamawaki T, Yamada K: Effect of subthalamic deep brain stimulation on postural abnormality in Parkinson disease. J Neurosurg 112:1283-1288, 2010

49. Upadhyaya CD, Starr PA, Mummaneni PV: Spinal deformity and Parkinson disease: a treatment algorithm. Neurosurg Focus 28(3):E5, 2010

50. Wadia PM, Tan G, Munhoz RP, Fox SH, Lewis SJ, Lang AE: Surgical correction of kyphosis in patients with camptocormia due to Parkinson's disease: a retrospective evaluation. J Neurol Neurosurg Psychiatry 82:364-368, 2011

51. Wrede A, Margraf NG, Goebel HH, Deuschl G, SchulzSchaeffer WJ: Myofibrillar disorganization characterizes myopathy of camptocormia in Parkinson's disease. Acta Neuropathol 123:419-432, 2012

52. Yamada K, Shinojima N, Hamasaki T, Kuratsu J: Subthalamic nucleus stimulation improves Parkinson's disease-associat- ed camptocormia in parallel to its preoperative levodopa responsiveness. J Neurol Neurosurg Psychiatry 87:703-709, 2016

\section{Disclosures}

Dr. Larson reports receipt of honoraria from Medtronic and support of non-study-related clinical or research effort from Voyager Therapeutics and serving as a consultant for Abbott. Dr. Mummaneni reports a consultant relationship with DePuy Spine, Globus, and Stryker; direct stock ownership in Spinicity/ISD; support of non-study-related clinical or research effort from NREF and ISSG; royalties from DePuy Spine, Thieme, and Springer; and honoraria from AO Spine.

\section{Author Contributions}

Conception and design: AK Chan, Mummaneni. Acquisition of data: AK Chan, AY Chan. Analysis and interpretation of data: AK Chan, AY Chan, Mummaneni. Drafting the article: all authors. Critically revising the article: all authors. Reviewed submitted version of manuscript: AK Chan, Lau, Durcanova, Miller, Larson, Starr, Mummaneni. Approved the final version of the manuscript on behalf of all authors: AK Chan. Statistical analysis: AK Chan, AY Chan, Mummaneni. Administrative/technical/ material support: Miller. Study supervision: Mummaneni.

\section{Supplemental Information}

Current Affiliations

Alvin Y. Chan: Department of Neurological Surgery, University of California, Irvine, Orange, CA.

\section{Correspondence}

Andrew K. Chan: University of California, San Francisco, CA. andrew.chan@ucsf.edu. 